Introduction Elderly patients are less likely to receive radical treatment for lung cancer. Palma et al (2010) demonstrated an increase in radical treatment rates for patients aged $\geq 75$ years in Holland following the introduction of Stereotactic Ablative Radiotherapy (SABR), without reduction in surgical resections. This was associated with improved overall survival. There are international differences in radical treatment rates and outcomes in lung cancer. We aim to evaluate the changes in lung cancer treatment and outcomes following the introduction of SABR in the UK.

Methods This is a retrospective observational study at a large UK teaching hospital. Data for patients diagnosed over seven years (2008-2014) were analysed from a local dataset maintained for the National Lung Cancer Audit. SABR was introduced for lung cancer in Leeds in 2010. Statistical analyses were performed using Chi-square, t-test and Kaplan Meier survival analysis.

Results There were 1874 new diagnoses of lung cancer in patients aged $\geq 75$ years, accounting for $45.3 \%$ of all new diagnoses. Comparing patients $\geq 75$ years pre-SABR (20082009) and post-SABR (2011-2014), there was an increase in the proportion of early stage disease (stage I-IIA $22.5 \%$ to $29.2 \%, \mathrm{p}=0.0054)$. Of the 502 patients with early stage disease, there was no change in performance status (PS 0\%-2 $68.4 \%$ to $63.3 \%, \mathrm{p}=0.2468$ ) or age at diagnosis (median (IQR) 81.1 (78.0-84.3) to $80.9(77.7-85.1)$ years, $\mathrm{p}=0.7422)$. Rates of radical radiotherapy/SABR $(12.2 \%$ to $39.2 \%$, $\mathrm{p}<0.001)$ have increased, while surgical resections $(28.9 \%$ to $28.5 \%, \mathrm{p}=1.000$ ) have remained stable and the proportion of patients receiving palliative treatment/best supportive care (BSC) has decreased $(58.8 \%$ to $32.3 \%, \mathrm{p}<0.001)$ (figure 1). Median overall survival has increased (518 to 687 days, $\mathrm{p}=0.0016$ ).

Discussion The proportion of elderly patients being diagnosed with early stage lung cancer is increasing. There has been no significant change in the demographics of those with early stage disease. Following the introduction of SABR in 2010, there has been an increase in radical radiotherapy treatment for elderly patients with early stage disease, with no sustained change in surgical resection rates and increase in overall rates of radical treatment. This was associated with a significant improvement in overall survival.

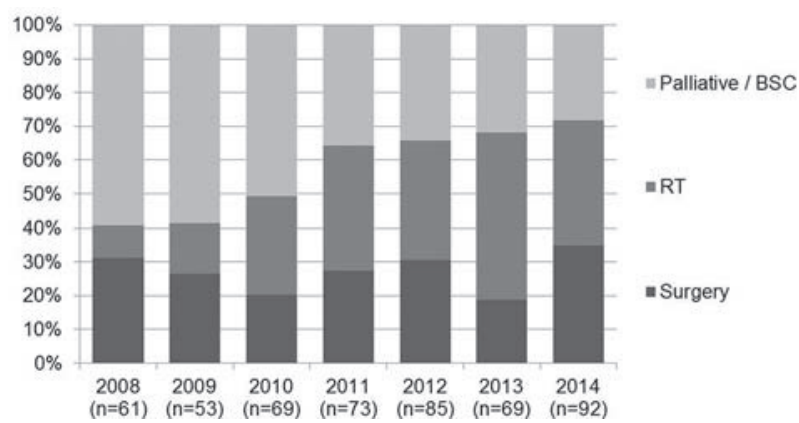

Abstract 597 Figure 1 Treatment of stage I-IIA lung cancer in patients $\geq 75$ years.

\section{S98 TREATMENT PATTERNS AND SURVIVAL OUTCOMES OF STAGE IIIA (N2) NON -SMALL CELL LUNG CANCER IN ENGLAND}

${ }^{1} \mathrm{JB}$ Adizie, ${ }^{2} \mathrm{~A}$ Khakwani, ${ }^{2} \mathrm{P}$ Beckett, ${ }^{2} \mathrm{~N}$ Navani, ${ }^{2} \mathrm{~S}$ Harden, ${ }^{2} \mathrm{~W}$ Woolhouse. ${ }^{1}$ Heart of England NHS Foundation Trust, Birmingham, UK; ${ }^{2}$ Care quality improvement department, Royal College of Physicians, London, UK

\subsection{6/thoraxjnl-2017-210983.104}

Introduction The optimal management of lung cancer patients with metastatic involvement of the ipsilateral mediastinum (N2 disease) remains controversial. Randomised controlled trials have failed to demonstrate superiority of one bimodality strategy over another (chemotherapy plus surgery versus chemotherapy plus radiotherapy). There is little knowledge of real world experience of the uptake of different treatment regimens and the corresponding survival outcomes. Data collected via the National Lung Cancer Audit (NLCA) and linked for the first time to the national radiotherapy dataset (RTDS) allow us to describe the treatment patterns and outcomes of patients with N2 disease in England.

Methods Patients diagnosed with stage T1-3, N2, M0 nonsmall cell lung cancer between 1 st January 2015 and 31 st December 2015 were identified. The dose and schedule of radiotherapy treatments described in the RTDS were used to determine if the radiotherapy was given with radical or palliative intent. The proportion of patients alive at the time of data analysis (9-21 months from diagnosis) were calculated according to treatment category.

Results 2305 of 36025 (6.4\%) patients met the inclusion criteria. The proportion of patients receiving each treatment

Abstract S98 Table 1 Treatment patterns and survival outcomes of stage 3a (N2) lung cancer ( $n=2305)$

\begin{tabular}{llll}
\hline Groups & $\begin{array}{l}\text { Number } \\
(\mathbf{n})\end{array}$ & $\begin{array}{l}\text { Percentage } \\
(\%)\end{array}$ & $\begin{array}{l}\text { Survival } \\
(\%)\end{array}$ \\
\hline Group 1: Surgery & 450 & 19.5 & 74.4 \\
Surgery & 165 & 7.2 & 61.8 \\
Surgery and Adjuvant Chemotherapy & 222 & 9.6 & 82.9 \\
Neoadjuvant chemotherapy and surgery & 21 & 0.9 & 90.5 \\
Surgery and Radical Radiotherapy & 5 & 0.2 & 60.0 \\
Triple therapy & 37 & 1.6 & 73.0 \\
& & & \\
Group 2: Radical Radiotherapy & 435 & 18.9 & 63.2 \\
Radical Radiotherapy & 205 & 8.9 & 61.5 \\
Radical Radiotherapy and Chemotherapy & 230 & 10.0 & 64.8 \\
& & & \\
Group 3: Palliative intent treatment & 618 & 26.8 & 41.8 \\
Palliative radiotherapy and chemotherapy & 142 & 6.2 & 36.6 \\
Palliative radiotherapy & 249 & 10.8 & 29.7 \\
Palliative Chemotherapy alone & 227 & 9.8 & 58.2 \\
Group 4: Best Supportive Care (No & 802 & 34.8 & 23.1 \\
treatment) & & & \\
\hline
\end{tabular}


modality with corresponding survival are shown in Table 1. $243(10.5 \%)$ patients received surgery and chemotherapy, 230 $(10 \%)$ patients received radical radiotherapy and chemotherapy, $618(26.8 \%)$ palliative radiotherapy or palliative chemotherapy and $802(34.8 \%)$ received best supportive care. The proportion of patients alive was $74.4 \%$ in patients receiving surgery; $63.2 \%$ for patients receiving radical radiotherapy, $41.8 \%$ for palliative chemotherapy/radiotherapy and $23.1 \%$ for supportive care.

Conclusions The commonest curative intent treatments are bimodality treatment (chemotherapy combined with either surgery or radical radiotherapy), however only one fifth of patients received this. The majority of patient still receive palliative treatment only. Survival is higher in patients who receive surgery as part of their treatment however we are unable to exclude selection bias as the reason for this. Further risk adjustment analysis will be performed to assess this.

\section{S99 LUNG CANCER SURGICAL OUTCOMES IN ENGLAND (2015)}

${ }^{1} \mathrm{P}$ Beckett, ${ }^{1} \mathrm{R}$ Dickinson, ${ }^{2} \mathrm{R}$ Hubbard, ${ }^{2} \mathrm{~A}$ Khakwani, ${ }^{3} \mathrm{D}$ West. ${ }^{1}$ Royal College of Physicians, London, UK; ${ }^{2}$ University of Nottingham, Nottingham, UK; ${ }^{3}$ Society of Cardiothoracic Surgeons, London, UK

\subsection{6/thoraxjnl-2017-210983.105}

Introduction The National Lung Cancer Audit has collected data for over 10 years demonstrating gradually rising resection rates in the UK. The Clinical Outcomes Programme (COP) uses national audit data to publish quality measures at the level of individual consultants. The lung cancer COP focusses on activity at individual surgeon level, and on survival at unit level. Previous rounds of this audit demonstrated overall 30

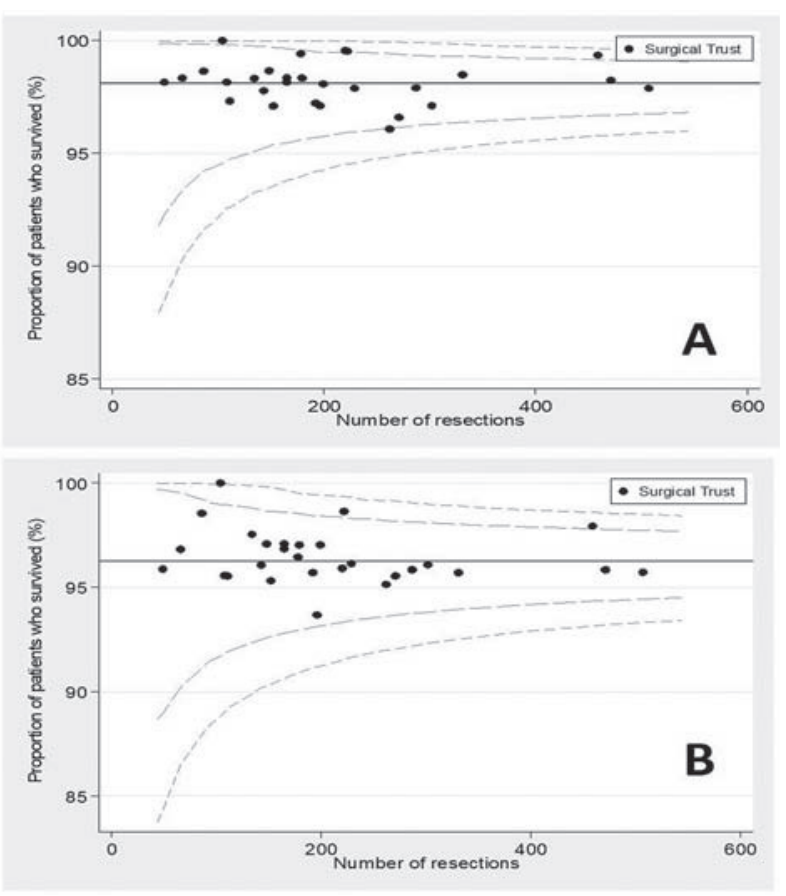

Abstract S99 Figure 1 Funnel plots of adjusted survival for 30 days (A) and 90 days (B) survival after surgery for lung cancer in English trusts. and 90 day survival of $97.8 \%$ and $95.5 \%$ (2013) and $97.9 \%$ and $96.2 \%$ (2014).

Methods Data submitted to the NLCA for patients having curative-intent surgery who underwent surgery in 2015 was sent to the clinical lead at each surgical unit for validation and addition of responsible surgeon GMC number, with the option to add surgical cases if they were not included in the supplied dataset. Date of death was derived by a link to the Office of National Statistics. Units reporting unadjusted survival proportions more than three standard errors outside the national mean ("alarm" level) at 30 or 90 days were identified as statistical outliers.

Results All of the 28 surgical units in England participated in the audit, submitting a total of 5843 cases, of which $93 \%$ were NSCLC, 2\% SCLC and 6\% carcinoid. Median annual unit activity was 186 resections (IQR 141-264, range 49507), an increase over the median 156 in the previous year. Median annual activity for individual surgeons was 44 (IQR 18-67, range 1-171). Median LOS 8.1 days (median 6, IQR 4-9). Overall 30 day survival was $98.2 \%$ and 90 day survival was $96.3 \%$. There were no units with statistical outliers at the alarm level at 30 days and 90 days (see figure 1). Overall 1 year survival $87.9 \%$.

Conclusion Volume of activity varies widely by unit and individual surgeon. Survival after lung cancer surgery is very high, is improving, and is not statistically significantly different across the surgical units in England. New measures are needed to properly reflect features that are important to patients.

\section{S100 UTILITY OF ENDOBRONCHIAL ULTRASOUND-GUIDED TRANSBRONCHIAL NEEDLE ASPIRATION FOR PD-L1 TESTING IN PATIENTS WITH NSCLC}

${ }^{1} \mathrm{~F}$ Perrotta, ${ }^{2} \mathrm{~B}$ Adizie, ${ }^{3} \mathrm{U}$ Maqsood, ${ }^{4} \mathrm{M}$ Elshafi, ${ }^{5} \mathrm{~S}$ Jafri, ${ }^{2}$ I Woolhouse, ${ }^{3} \mathrm{M}$ Munawar, ${ }^{4} \mathrm{M}$ Evison, ${ }^{4} \mathrm{R}$ Booton, ${ }^{5} \mathrm{DR}$ Baldwin, ${ }^{6} \mathrm{SM}$ Janes, ${ }^{1} \mathrm{~A}$ Bianco, ${ }^{6} \mathrm{~N}$ Navani. ${ }^{1}$ University of Campania "L. Vanvitelli", Naples, Italy; ${ }^{2}$ University Hospitals Birmingham, Birmingham, UK; ${ }^{3}$ Lancashire Teaching Hospitals, Preston, UK; ${ }^{4}$ University Hospital of South Manchester, Manchester, UK; ${ }^{5}$ Nottingham University Hospitals, Nottingham, UK; ${ }^{6}$ University College of London, London, UK

\subsection{6/thoraxjnl-2017-210983.106}

Rationale Recent data have demonstrated the superiority of Pembroluzimab over chemotherapy for patients with advanced NSCLC and high ( $\geq 50 \%$ expression) of PD-L1.1 This has resulted in NICE approving Pembroluzimab as a first line treatment option for patients with advanced NSCLC in June 2017. The original trial however excluded patients with PDL1 testing on EBUS samples. We therefore conducted a large, multicentre study to clarify whether specimens obtained by EBUS-TBNA were suitable for testing PD-L1 in patients with NSCLC.

Methods NSCLC samples acquired by EBUS-TBNA (29.4\%), percutaneous biopsy (31.2\%), endobronchial biopsy (13.8\%), surgical $(21.4 \%)$ or other techniques $(4.1 \%)$ were recorded from 435 consecutive patients with known or suspected lung cancer across 5 centres in England between January 2015 and December 2016.

Results PD-L1 assessment (using the $22 \mathrm{C} 3$ assay in all cases) was possible in $92.2 \%$ of patients undergoing EBUS and there was no difference in success of PD-L1 testing according to modality of tissue acquisition $(p=0.18)$. The frequency of complications from EBUS-TBNA was similar to endobronchial or percutaneous techniques but lower than surgical procedures 